\title{
Density Functional Theory Study of the Adsorption of Oxygen and Hydrogen on 3d Transition Metal Surfaces with Varying Magnetic Ordering
}

\author{
Mogamat A. Peck $^{\mathrm{a}, *(\mathbb{D})}$, David Santos-Carballal ${ }^{\mathrm{b}, \mathrm{C}} \mathbb{D}$, Nora H. de Leeuw ${ }^{\mathrm{b}, \mathrm{c}} \mathbb{( D )}$ and Michael Claeys ${ }^{\mathrm{a}}$ (iD) \\ ${ }^{a}$ Catalysis Institute and c*change (DSI-NRF Centre of Excellence in Catalysis), Department of Chemical Engineering, \\ University of Cape Town, Private Bag X3, Rondebosch, 7701, South Africa. \\ ${ }^{b}$ School of Chemistry, Cardiff University, Main Building, Park Place, CF10 3AT, Cardiff, United Kingdom.

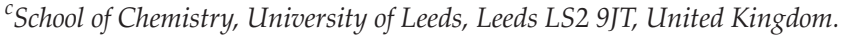

Received 15 April 2020, revised 5 November 2020, accepted 6 November 2020.

\begin{abstract}
We have employed density functional theory (DFT) calculations to investigate the adsorption of molecular oxygen and hydrogen on $3 \mathrm{~d}$ transition metal (TM) surfaces with varying ordered magnetic structures in the bulk, namely ferromagnetic Fe(110), $\mathrm{Co}(0001), \mathrm{Ni}(111)$ and diamagnetic $\mathrm{Cu}(111)$. The trend observed in the energies of adsorption was compared with the magnetic moment of the cell using the $d$-band centre model of chemisorption and the Stoner model of magnetic energy. As the gap between the d-band centre and the Fermi level of the TM decreases, more antibonding orbitals are present above the Fermi level and thus unoccupied, leading to stronger binding. Correspondingly, the shift in the d-band centre decreases the density of states (DOS) at the Fermi level giving rise to the ordered magnetic structure.
\end{abstract}

KEYWORDS

d-Band centre, chemisorption, Hedvall effect, magnetism.

\section{Introduction}

The interactions between gas molecules and transition metal (TM) surfaces are vital to understanding the underlying mechanics of heterogeneous catalysis. Thus, optimizing adsorption sites on TM surfaces has driven a variety of theoretical and experimental studies. ${ }^{1}$ It is well-understood that the interaction between an adsorbate and TM surface largely depends on the d-electrons of the metal surface. The most widely used descriptor of this interaction is the $d$-band centre model pioneered by Hammer and Nørskov. ${ }^{2}$ The d-band centre model, in principle, states that changes to adsorption energies over TM surfaces are related to the shifting of the metal's d-band centre with respect to its Fermi level. As shown in Fig. 1, the higher the shift of the d-band centre (i.e. smaller $\Delta \mathrm{E}_{\mathrm{F}}-\mathrm{E}_{\mathrm{dbc}}$ ), the lower the occupancy of the anti-bonding states, corresponding to a stronger TM-adsorbate binding energy. Bhattacharjee ${ }^{3}$ has shown that for magnetically polarized TM surfaces, distinct d-band centres should be considered for spin-up and spin-down states. These two d-band centres are shifted in opposite directions relative to the unpolarized d-band centre, resulting in competing contributions (i.e. attractive and repulsive) to the TM-adsorbate orbitals. The extent of this competition depends solely on the magnitude of the TM magnetic polarization. For the purposes of this study, the conventional d-band centre model was used, since the trends observed for the TM of interest would be similar, as shown by Bhattacharjee.

The work function (WF) of the metal is another important descriptor to consider during chemisorption. The WF is defined as the difference in energy between an electron in the surface and that of an electron removed to the vacuum ${ }^{4}$ and thus it can be associated with the minimum work required to the facilitate

\footnotetext{
* To whom correspondence should be addressed. E-mail: adli.peck@gmail.com
}

the electron transfer between the metal and the adsorbate. The lowest WF for transition metals are generally found on the least densely packed surface. ${ }^{5}$

Another important property of TM, which also largely depends on the d-electrons, ${ }^{6}$ is their magnetic ordering (i.e. paramagnetic, ferromagnetic, diamagnetic). The role of the ordered magnetic structure, temperature-dependent magnetic-order transitions and bulk magnetic domain formation during the chemisorption of adsorbates is less well-understood. This is further complicated by the variation in magnetic ordering of the TM bulk structure compared to the surface where chemisorption occurs. ${ }^{7}$

The magnetic moment of $3 \mathrm{~d}$ TM can be described by quantum formulations of electron spin and orbital contributions of

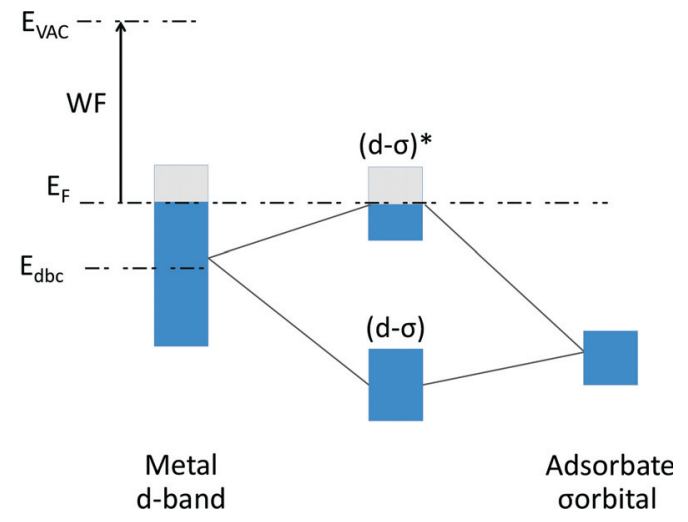

Figure 1 The metal d-band hybridizes with the bonding orbital of the adsorbate to form bonding and anti-bonding orbitals. The higher the $\mathrm{d}$-band centre $\left(\mathrm{E}_{\mathrm{dbc}}\right)$ relative to the Fermi level $\left(\mathrm{E}_{\mathrm{F}}\right)$ (i.e. smaller $\left.\Delta \mathrm{E}_{\mathrm{F}}-\mathrm{E}_{\mathrm{dbc}}\right)$ the stronger the metal-adsorbate binding energy. The work function (WF) is defined as the work required to move an electron from the metal surface to the vacuum region. 
individual atoms that comprise the bulk structure. ${ }^{8}$ Interestingly, spin-orbital coupling has been shown to make a negligible contribution to the atomic magnetic moments. ${ }^{9}$ This description, however, is difficult to relate to the chemisorption of adsorbates since the adsorption energy, as mentioned above, is easily described in terms of the d-band energy. A more useful descriptor to relate trends in chemisorption with magnetic ordering is the energy band theory of magnetism (or Stoner Mean-Field theory). ${ }^{10}$ In the Stoner model, the magnetically ordered structure is described in terms of the DOS at the Fermi level, $\mathrm{D}\left(\mathrm{E}_{\mathrm{F}}\right)$, and an exchange integral parameter (or Stoner parameter, $I$ ) which in turn depends on the number of d-electrons. The Stoner criterion, described as $\mathrm{D}\left(\mathrm{E}_{\mathrm{F}}\right) \mathrm{I}>1$, is a measure by which the ferromagnetic ordering of a TM can be determined. As shown in Fig. 2, the $\mathrm{D}\left(\mathrm{E}_{\mathrm{F}}\right)$ for the $3 \mathrm{~d}$ TM increases from $\mathrm{Mn}$ to $\mathrm{Ni}$; however, only $\mathrm{Fe}, \mathrm{Co}$ and $\mathrm{Ni}$ exceed the Stoner criterion.

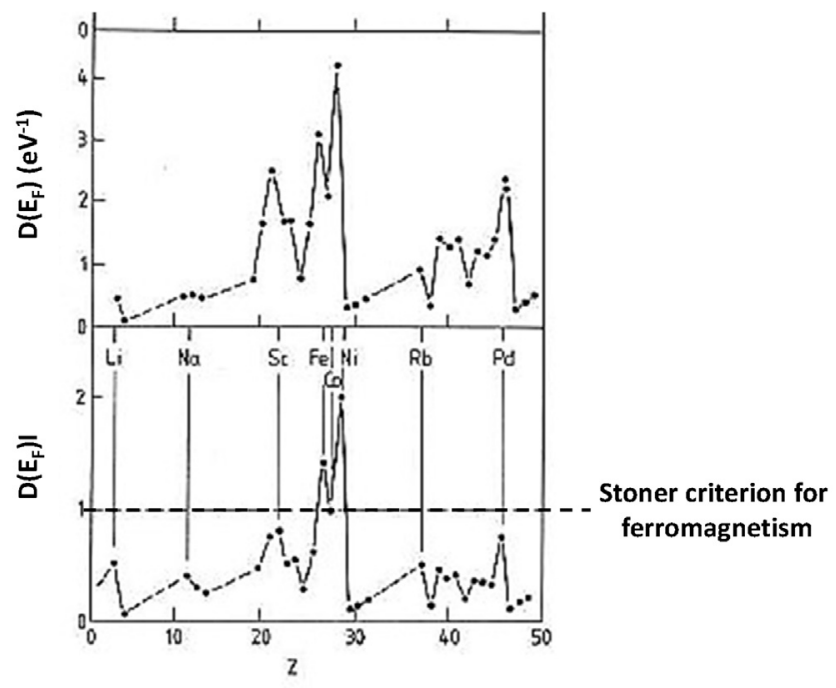

Figure 2 The trend over all elements for the DOS at the Fermi level, D( $\left.E_{F}\right)$, (top). The Stoner criterion for ferromagnetic ordering is obtained when $\mathrm{D}\left(\mathrm{E}_{\mathrm{F}}\right) \mathrm{I}>1$, where $\mathrm{I}$ is the Stoner parameter. ${ }^{11}$

The role of TM magnetic ordering in the kinetics and thermodynamics of chemisorption is crucial to the understanding of the magneto-catalytic effect. ${ }^{12}$ The magneto-catalytic (or Hedvall) effect is a contested theory based on early experimental and theoretical studies, which suggests that the magnetic-ordering of a metal can influence the catalytic properties of a reaction. The initial experimental studies were conducted by Hedvall, ${ }^{13}$ showing that the activity of nickel-catalysts for the decomposition of $\mathrm{N}_{2} \mathrm{O}$ could be manipulated by varying the temperature-dependent magnetic ordering of the bulk structure. Early theoretical work conducted by Ilisca ${ }^{14}$ and Selwood ${ }^{15}$ focused on the application of magnetic fields for ortho-para hydrogen conversions. A handful of theoretical works ${ }^{16}$ have surfaced in recent years on the role of magnetism in catalysis, which provide further evidence of Hedvall's theory.

Melander et al. ${ }^{17}$ computed the adsorption of $\mathrm{H}_{2}$ and $\mathrm{CO}$ on face centred cubic $(f c c) \mathrm{Fe}(111)$ surfaces with different magnetic configurations, namely ferromagnetic and antiferromagnetic structures. The hypothetical variation of the magnetic ordering results in the orbital occupancy of the surface differing due to the variation of the spin structures. The molecular adsorption study indicates that there is charge transfer between the iron surface and either adsorbate, which is greater for the bilayer system with the antiferromagnetic order than with the ferromagnetic configuration. In addition, the dissociation of $\mathrm{H}_{2}$ is easier on the bilayer with the antiferromagnetic order, whereas $\mathrm{CO}$ dissociation is more difficult on the ferromagnetic surface.

The influence of magnetic ordering in surfaces on the molecular adsorption behaviour is also observed when the adsorbate has a net magnetic moment, as is the case for oxygen. During the oxygen evolution reaction, the spin conversion from singlet-state oxygen in water to triplet state molecular oxygen is considered the rate-limiting step for water splitting. This is a spin-forbidden isogyric reaction, where a heterogeneous catalyst is needed to allow non-conserving spin reactions. For example, Lim et al. ${ }^{18}$ showed that the antiferromagnetic ordering of perovskite catalysts was important for facilitating this conversion. Similarly, Torun et al. ${ }^{19}$ showed that the presence of magnetic moments in the RuO2 (110) surface facilitates the selection rule for the spin conversion and evolution of the triplet state molecular oxygen. This spin conversion can be further amplified by weak magnetic fields $(<0.4 \mathrm{~T})$ applied to the magnetic anodes of alkaline water electrolysis. ${ }^{20}$

In this study the adsorption properties of selected 3d-TM surfaces with varying magnetic ordering is compared using molecular oxygen and hydrogen. Density functional theory (DFT) calculations are used to model the magnetic structures (i.e. non-paramagnetic ordering) and molecular adsorptions to the surfaces. Iron, cobalt and nickel are ferromagnetic materials, while copper is diamagnetic. The bcc-Fe(110), hcp-Co(0001), fcc-Ni(111) and $\mathrm{Cu}(111)$ surfaces are investigated since these planes are essentially the same or (as in the case of $\mathrm{Fe}$ ) similar, thus minimizing the contribution of varying lattice

\section{Methodology}

Spin-polarized DFT calculations were performed using the Perdew-Burke-Ernzerhof (PBE) exchange correlation functional, ${ }^{21}$ within the generalized gradient approximation (GGA). The Vienna Ab Initio Simulation Package (VASP ${ }^{22}$ was employed for the optimizations of the atomic positions and lattice parameters of the metal bulk phases and the position of the exposed atoms in the surfaces. An electronic step convergence of $10^{-5} \mathrm{eV}$ was used, along with a tight atomic force threshold of $0.01 \mathrm{eV} / \AA$. The projector augmented wave pseudopotentials were used to treat the core electrons and their effect on the valence electrons. ${ }^{23}$ A plane-wave basis set has been used with a kinetic energy limit of $400 \mathrm{eV}$ and a Monkhorst Pack k-points mesh of $7 \times 7 \times 1$ to sample the Brillouin zone. Dipole corrections were applied in the direction perpendicular to the surface. The formalism of Grimme with the Becke-Johnson damping (D3-BJ) was applied to account for the long-range dispersion corrections. ${ }^{24}$ The Methfessel-Paxton method order of 1 was used to determine the electronic partial occupancies with the width of the smearing set at $0.02 \mathrm{eV}$. To simulate the $\mathrm{Fe}(110), \mathrm{Co}(0001), \mathrm{Ni}(111)$ and $\mathrm{Cu}(111)$ surfaces, a set of slab models were constructed using $(1 \times 1)$ supercells keeping a vacuum region of $10 \AA$ normal to the surface. The energy of adsorption $\left(\mathrm{E}_{\mathrm{ads}}\right)$ of a single oxygen molecule in the triplet spin state or hydrogen molecule in equilibrium with a reservoir of oxygen or hydrogen, respectively, was calculated according to:

$$
\mathrm{E}_{\mathrm{ads}}=\mathrm{E}_{\text {tot }}-\mathrm{E}_{\mathrm{M}}-\frac{1}{2} \mathrm{E}_{\mathrm{X}_{2}}
$$

where $E_{\text {tot }}$ is the total energy of the TM-adsorbate system, $E_{M}$ is the optimized energy of the TM surface and $E_{X_{2}}$ is the energy of a single $\mathrm{O}$ or $\mathrm{H}$ atom in the diatomic $\mathrm{H}_{2}$ or $\mathrm{O}_{2}$ molecules, respectively, accounting for over-binding in the case of $\mathrm{O}_{2}$. The magnetic moment of the cell was also obtained and correlated with the energy of adsorption for each TM adsorption. 


\section{Discussion}

Both hydrogen and oxygen adsorb dissociatively to the metal surfaces. The adsorption energy of oxygen (see Fig. 3 bottom panel) is lower than hydrogen across most surfaces with the largest difference observed for $\mathrm{Co}(001)$. The trend across the metals agree previous literature reports, ${ }_{1}^{1 \mathrm{~b}, 25 b, 26}$ generally growing more positive from iron to copper. The magnetic moment of the cell for each metal-adsorbate is inversely proportional to the adsorption energies, decreasing from iron to copper.

The trends observed in the adsorption energies and magnetic moments can both be explored as functions of the DOS at the Fermi level. As the number of d-electrons increases from iron to copper, the d-band centre shifts further from the Fermi level (increasing $\Delta \mathrm{E}_{\mathrm{F}}-\mathrm{E}_{\mathrm{dbc}}$ ) leading to the strongest and weakest adsorption energies, respectively (Fig. 4). As discussed above, using the Stoner Mean Field theory, the DOS at the Fermi level determines the magnetic ordering of the TM. As the adsorption energy decreases the local DOS at the Fermi level, a reduction in the local magnetic moment is also observed.

This study has sought to explore the trends observed in chemisorption and magnetic ordering from a common fundamental property of TM. An inverse relationship between chemisorption and magnetic ordering was observed for the $3 \mathrm{~d}$ TM, which can both be rationalized using theories founded on the DOS at the Fermi level. This is a useful basis from which to understand experimental theories of magneto-catalysis currently under investigation.
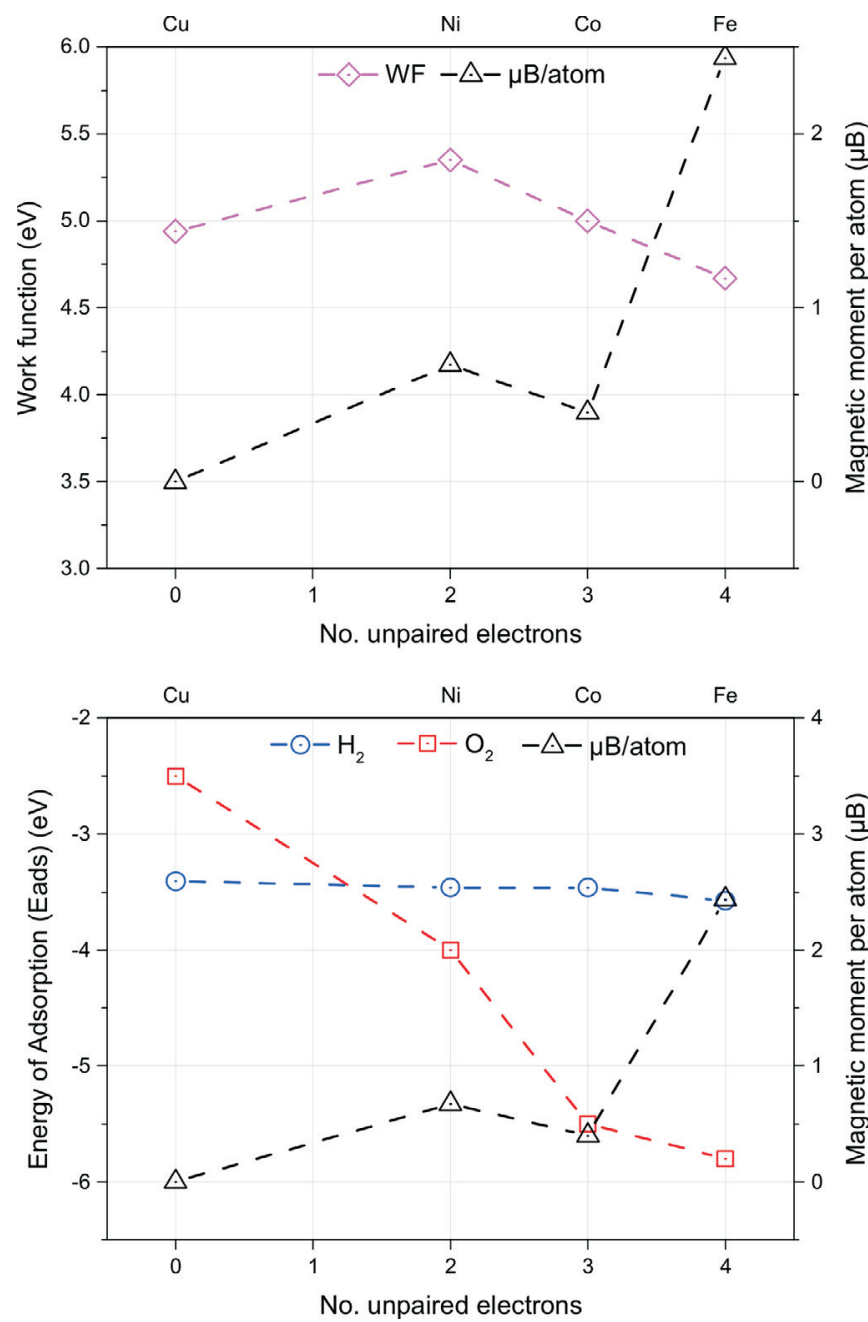

Figure 3 Trends in (top) the work function ${ }^{4-5}$ and (bottom) magnetic moment per atom and adsorption energy of oxygen and hydrogen..$^{25}$

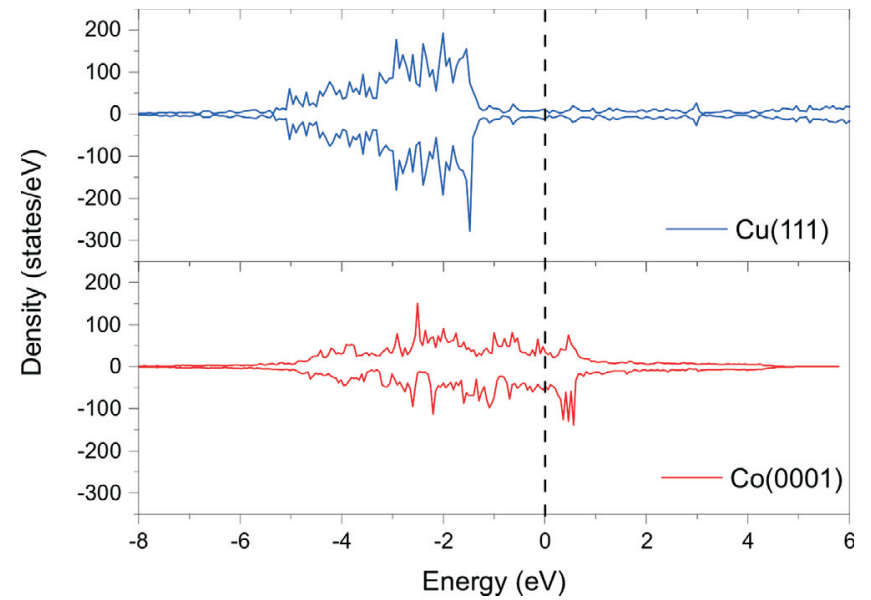

Figure 4 DOS for fcc-Cu(111) and hcp-Co(0001), dashed line indicates the Fermi level.

\section{Acknowledgements}

We acknowledge funding of a Newton UK-South Africa International PhD Partnering programme by the UK Economic and Social Research Council (grant no. ES/N013867/1) and the National Research Foundation, South Africa. We further acknowledge use of computational resources of the Centre for High Performance Computing (CHPC) of South Africa; Advanced Research Computing@ Cardiff (ARCCA) Division, Cardiff University; and HPC Wales, and associated support services, in the completion of this work. We thank Prof. Eric Steen for his support and we are also grateful for support received by the National Research Foundation (NRF), the DSI-NRF Centre of Excellence in Catalysis ( $c^{*}$ change) and a Sasol University Research Grant.

\section{${ }^{5}$ ORCID iDs}

M.A. Peck:

D. Santos-Carballal:

N.H. de Leeuw:

M. Claeys:

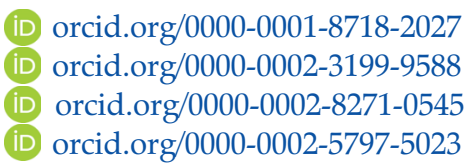

\section{References}

1 J. Nørskov, Chemisorption on metal surfaces, Rep. Prog. Phys., 1990, 53, 1253; (b) I. Toyoshima and G.A. Somorjai, Heats of chemisorption of $\mathrm{O} 2, \mathrm{H} 2, \mathrm{CO}, \mathrm{CO} 2$, and $\mathrm{N} 2$ on polycrystalline and single crystal transition metal surfaces, Catal. Rev., 1979, 19, 105-159.

2 (a) B. Hammer and J. Nørskov, Electronic factors determining the reactivity of metal surfaces, Surf. Sci., 1995, 343, 211-220; (b) B. Hammer, J.K. Nørskov, in Advances in Catalysis, vol. 45, Elsevier, 2000, pp. 71-129

3 S. Bhattacharjee, U.V. Waghmare and S.-C. Lee, An improved d-band model of the catalytic activity of magnetic transition metal surfaces, Sci. Reports, 2016, 6, 35916.

4 D.R. Lide, CRC Handbook of Chemistry and Physics, 85th edn., Taylor \& Francis, 2004

5 N. Lang and W. Kohn, Theory of metal surfaces: work function, Phys. Rev. B, 1971, 3, 1215.

6 P. Brown, Magnetism of ferromagnetic metals above their Curie temperature, Europhys. News, 1986, 17, 25-28.

7 M. Petersen, J. Hafner and M. Marsman, Structural, electronic and magnetic properties of Gd investigated by DFT + U methods: bulk, clean and H-covered (0001) surfaces, J. Phys.: Condens. Matter, 2006, 18, 7021

8 Y.A. Izyumov, in AIP Conference Proceedings, vol. 678, AIP, 2003, pp. $181-272$.

9 A. Roldan, D. Santos-Carballal and N.H. de Leeuw, A comparative DFT study of the mechanical and electronic properties of greigite Fe3S4 and magnetite Fe3O4, J. Chem. Phys., 2013, 138, 204712.

10 (a) J.C. Slater, Energy-band theory of magnetism, J. Appl. Phys., 1968, 
39,761-767; (b) P. Marcus and V. Moruzzi, Stoner model of ferromagnetism and total-energy band theory, Phys. Rev. B, 1988, 38, 6949.

$11 \mathrm{H}$. Ibach and H. Lüth, Solid-state Physics: an Introduction to Theory and Experiment, Springer Science \& Business Media, 2012.

12 P. Selwood, Magnetism and catalysis, Chem. Rev., 1946, 38, 41-82.

13 G. Cohn and J. Hedvall, The influence of the transition from ferromagnetism to paramagnetism on catalytic activity, J. Phys. Chem., $1942,46,841-847$.

14 (a) E. Ilisca, Introduction to a theory of ortho-para $\mathrm{H} 2$ conversion on paramagnetic catalysts: the magnetic field effect, Phys. Rev. Lett., 1978, 40, 1535; (b) E. Ilisca and A. Legrand, Theoretical rates and correlation functions in ortho-para $\mathrm{H} 2$ conversion on paramagnetic surfaces, Phys. Rev. B, 1972, 5, 4994.

15 (a) P. Selwood, Magnetic field effects on the catalyzed nondissociative parahydrogen conversion rate, J. Catal., 1977, 50, 15-23; (b) P. Selwood, The effect of a weak magnetic field on the rare earth catalyzed parahydrogen conversion rate, J. Catal., 1971, 22, 123-129.

16 (a) J. Gracia, J. Munarriz, V. Polo, R. Sharpe, Y. Jiao, J. Niemantsverdriet and T. Lim, Analysis of the magnetic entropy in oxygen reduction reactions catalysed by manganite perovskites, ChemCatChem, 2017, 9, 3358-3363; (b) J. Gracia, R. Sharpe and J. Munarriz, Principles determining the activity of magnetic oxides for electron transfer reactions, J. Catal., 2018, 361, 331-338; (c) R. Sharpe, J. Munarriz, T. Lim, Y. J. Niemantsverdriet, V. Polo and J. Gracia, Orbital physics of perovskites for the oxygen evolution reaction, Top. Catal., 2018, 61, 267-275.

17 M. Melander, K. Laasonen and H. Jónsson, Effect of magnetic states on the reactivity of an FCC (111) iron surface, J. Phys. Chem. C, 2014, 118, 15863-15873.

18 T. Lim, J. Niemantsverdriet and J. Gracia, Layered antiferromagnetic ordering in the most active perovskite catalysts for the oxygen evolution reaction, ChemCatChem, 2016, 8, 2968-2974.

19 E. Torun, C. Fang, G. De Wijs and R. De Groot, Role of magnetism in catalysis: RuO2 (110) surface, J. Phys. Chem. C, 2013, 117, 6353-6357.
20 F.A. Garcés-Pineda, M. Blasco-Ahicart, D. Nieto-Castro, N. López and J.R. Galán-Mascarós, Direct magnetic enhancement of electrocatalytic water oxidation in alkaline media, Nature Energy, 2019, 4, $519-525$.

21 J.P. Perdew, K. Burke and M. Ernzerhof, Generalized gradient approximation made simple, Phys. Rev. Lett., 1996, 77, 3865.

22 G. Kresse and J. Furthmüller, Efficient iterative schemes for ab initio total-energy calculations using a plane-wave basis set, Phys. Rev. B, 1996, 54, 11169 .

23 P.E. Blöchl, Projector augmented-wave method, Phys. Rev. B, 1994, 50, 17953.

24 (a) S. Grimme, J. Antony, S. Ehrlich and H. Krieg, A consistent and accurate $\mathrm{ab}$ initio parametrization of density functional dispersion correction (DFT-D) for the 94 elements H-Pu, J. Chem. Phys., 2010, 132, 154104; (b) S. Grimme, S. Ehrlich and L. Goerigk, Effect of the damping function in dispersion corrected density functional theory, J. Comput. Chem., 2011, 32, 1456-1465.

25 (a) A. Nilsson, L. Pettersson, B. Hammer, T. Bligaard, C.H. Christensen and J.K. Nørskov, The electronic structure effect in heterogeneous catalysis, Catal. Lett., 2005, 100, 111-114; (b) P. Nordlander, S. Holloway and J. Nørskov, Hydrogen adsorption on metal surfaces, Surf. Sci., 1984, 136, 59-81.

26 (a) D.C. Sorescu, First principles calculations of the adsorption and diffusion of hydrogen on $\mathrm{Fe}(100)$ surface and in the bulk, Catal. Today, 2005, 105, 44-65; (b) S. Liem, J. Clarke and G. Kresse, Dissociation pathways of oxygen on copper (110) surface: a first principles study, Comput. Mater. Sci., 2000, 17, 133-140; (c) H.J. Elmers and U. Gradmann, Surface magnetism of oxygen and hydrogen adsorption on Ni(111), J. Appl. Phys., 1988, 63, 3664-3666; (d) A. Soon, M Todorova, B. Delley and C. Stampfl, Oxygen adsorption and stability of surface oxides onCu(111): a first-principles investigation, Phys. Rev. B, 2006, 73; (e) D. Klinke Ii and L.J. Broadbelt, A theoretical study of hydrogen chemisorption on $\mathrm{Ni}$ (111) and $\mathrm{Co}$ (0001) surfaces, Surf. Sci., 1999, 429, 169-177. 